20

\title{
Визуализация кровотока методом лазерных спекл-контрастных измерений в условиях неэргодичности
}

\author{
() А.Ю. Сдобнов ${ }^{1}$, В.В. Кальченко ${ }^{2}$, А.В. Быков ${ }^{1}$, А.П. Попов ${ }^{1}$, Г. Молодый ${ }^{2}$, И.В. Меглинский ${ }^{1,3,4,5,6,9}$ \\ ${ }^{1}$ University of Oulu, Optoelectronics and Measurement Techniques Laboratory, \\ Oulu 90570 Finland \\ ${ }^{2}$ Weizmann Institute of Science, Department of Veterinary Resources, \\ Rehovot 76100, Israel \\ ${ }^{3}$ Междисциплинарная лаборатория биофотоники, \\ Национальный исследовательский Томский государственный университет, \\ 634050 Томск, Россия \\ ${ }^{4}$ Инженерно-фризический институт биомедицины (ИФИБ), \\ Национальный исследовательский ядерный университет (МИФИ), \\ 115409 Москва, Россия \\ ${ }^{5}$ Aston Institute of Materials Research, School of Engineering and Applied Science, Aston University, \\ Birmingham, B4 7ET, UK \\ ${ }^{6}$ School of Life and Health Sciences, Aston University, \\ B4 7ET Birmingham, UK \\ e-mail: anton.sdobnov@oulu.fi, i.meglinski@aston.ac.uk
}

Поступила в редакцию 10.12.2019 г.

В окончательной редакции 03.02.2020 г.

Принята к публикации 28.02.2020 г.

Исследовано влияние неподвижных структурных включений в неоднородных сильно рассеивающих свет средах, таких как биоткани, на результаты спекл-контрастных измерений с использованием временного и пространственного методов обработки изображений методом лазерной спекл-контрастной визуализации. Детализированы границы применимости метода лазерной спекл-контрастной визуализации при невыполнении условия эргодичности. На базе модельных экспериментов показано, что увеличение в измеряемом объеме числа неподвижных рассеивателей по отношению к динамическим вносит существенную погрешность в результаты пространственной и временной обработки спекл-изображений при заданном времени экспозиции детектора. В то же время анализ пространственного и временного спекл-контрастов, значений коэффициента спекл-динамики, а также результаты моделирования измеряемого объема методом Монте-Карло показали, что наличие относительно тонкого, до $30 \%$ от общего объема, статически неподвижного слоя не вносит значительных изменений в результаты измерений методом лазерной спекл-контрастной визуализации. Время экспозиции камеры, а также количество кадров, используемых для обработки изображения, могут варьировать и быть подобраны индивидуально для каждого эксперимента. Разработанные алгоритмы пространственной и временной обработки изображений, полученных методом лазерной спекл-контрастной визуализации, были апробированы в экспериментах транскраниальной визуализации кровотока мозга мыши.

Ключевые слова: рассеяние, лазерное излучение, метод лазерной спекл-контрастной визуализации, эргодичность, неинвазивная визуализация, кровоток, мозг.

DOI: $10.21883 /$ OS.2020.06.49410.35-20

\section{Введение}

Метод лазерной спекл-контрастной визуализации (ЛСКВ) является сравнительно простым и весьма перспективным для исследования морфологических и функциональных изменений кровотока и перфузии биологических тканей in vivo [1]. Основу метода составляет статистический анализ интерференционных спекл-картин, образующихся на поверхности случайно-неоднородной мутной среды в результате рассеяния лазерного излучения. ЛСКВ успешно используется для визуализации кровотока и лимфотока в опухолевых тканях [2-4], оценки влияния аллергенов и оптических просветляющих агентов на микроциркуляцию крови в коже $[5,6]$, мониторинга мозгового кровотока [7,8], мониторинга перфузии кожи [9] и др. Преимуществами ЛСКВ являются возможность визуализации кровотока и перфузии биологических тканей в реальном времени, возможность проведения неинвазивных измерений, а также относительная дешевизна экспериментальной установки.

Классическая теория формирования спеклов подробно описана в работе [10]. Фактически лазерные спеклы являются случайной интерференционной картиной, возникающей вследствие взаимодействия когерентного света с рассеивающей поверхностью и/или с мутной негомогенной средой. В условиях экспериментального наблюдения изображение, формирующееся на каждом пикселе фотодетектора (ПЗС или КМОП каме- 
ра), является суперпозицией множества амплитудных функций рассеяния, каждая из которых возникает от различных точек рассеяния наблюдаемой среды. Таким образом, функции рассеяния имеют различные фазы, что и приводит к возникновению случайной интерференционной картины, получившей название спеклы или спекл-структуры [11]. Изменения локальной динамики в наблюдаемом объекте (движение рассеивающих частиц) приводит к флуктуациям спекл-структуры. Детектирование подобной спекл-структуры при помощи камеры с конечным временем экспозиции приводит к размытию получаемого спекл-изображения в областях, соответствующих движению рассеивающих частиц. Более того, чем интенсивнее движение в наблюдаемом объекте, тем более размытым будет спекл-изображение регистрируемое камерой. Статистический анализ спекл-структуры позволяет оценить среднюю скорость частиц в среде $[11]$.

Для подобной оценки был введен параметр - спеклконтраст $K[12]$ :

$$
K=\frac{\sigma}{\langle I\rangle},
$$

где $\langle I\rangle-$ средняя интенсивность спекл-структуры, $\sigma-$ стандартное отклонение интенсивности.

Теоретически $\langle I\rangle$ и $\sigma$ равны в случае идеально сформированной спекл-структуры. Из чего следует, что в данном случае значение спекл-контраста должно принимать значение 1. Тем не менее свойства используемой оптической системы, свойства самого наблюдаемого объекта, a также когерентные свойства лазерного источника влияют на формирование спекл-структуры, вследствие чего на практике спекл-контраст принимает значения между 0 и 1.

В работе [12] с помощью теории корреляционных функций была показана взаимосвязь между значением спекл-контраста, временем экспозиции камеры и временем спекл-корреляции. В работе [13] было предположено, что временные флуктуации интенсивности спеклструктуры, вызванные изменением электрического поля вследствие рассеяния света движущимися частицами, может быть связано с реальной скоростью этих частиц. Количественная оценка временных флуктуаций может быть осуществлена при помощи нормированной автокорреляционной функции электрического поля [14]:

$$
g_{1}(\tau)=\frac{\left\langle E(t) E^{*}(t+\tau)\right\rangle}{\left\langle E(t) E^{*}(t)\right\rangle},
$$

где $E(t)$ - электрическое поле детектируемого света и $E^{*}(t)$ - комплексное сопряжение электрического поля при времени $t, \tau-$ время задержки автокорреляции. Для оптических гауссовых случайных полей автокорреляционная функция (2) связана с автокорреляционной функцией интенсивности рассеянного света:

$$
g_{2}(\tau)=\frac{\langle I(t) I(t+\tau)\rangle}{\left\langle I(t)^{2}\right\rangle}
$$

Данная автокорреляционная функция второго порядка (3) может быть выражена через автокорреляционную функцию первого порядка (2) с помощью соотношения Зигерта [15]:

$$
g_{2}(\tau)=1+\beta\left|g_{1}(\tau)\right|^{2},
$$

где $\beta(0 \leq \beta \leq 1)$ является коэффициентом мультипликативного уменьшения спекл-контраста, связанного с такими факторами, как поляризация, эффекты когерентности и несоответствие между размером пикселя камеры и средним размером спекла [16].

Таким образом, уравнение (1) для спекл-контраста может быть представлено в виде

$$
K(T)=\left[\frac{2 \beta}{T} \int_{0}^{T}\left|\frac{g_{1}(\tau)}{g_{1}(0)}\right|^{2}\left(1-\frac{\tau}{T}\right) d \tau\right]^{1 / 2},
$$

где $K(T)$ является функцией спекл-контраста, зависящей от времени экспозиции камеры. В случае рассеяния на ансамбле статистически независимых частиц детектируемое поле представляет собой суперпозицию большого числа парциальных статистически независимых полей. В соответствии с центральной предельной теоремой статистика такого поля, является гауссовой, и только при этих условиях соотношение Зигерта (3) является действительным. Кроме того, описанная выше теория основана на предположении, что наблюдаемая среда является пространственно однородной, динамической и рассеянное от нее лазерное излучение удовлетворяет условию эргодичности $[17,18]$. На практике биологические ткани являются пространственно сильно неоднородными и состоят из динамических включений (кровь, лимфа) и из статических (кожа, кости и т.д.) компонент. Такая рассеивающая система не удовлетворяет условию эргодичности, что приводит к возникновению систематической ошибки метода ЛСКВ и, как следствие, к неверной интерпретации получаемых данных [16,19-21].

В ряде работ была рассмотрена возможность применения метода ЛСКВ в случае нарушений выполнения условия эргодичности [16,22-26]. Детектируемое лазерное излучение, рассеянное в биотканях, можно представить как сумму динамических $\left(E_{d}(t)\right)$ и статичных $\left(E_{s}\right)$ компонент электрического поля:

$$
E(t)=E_{d}(t)+E_{s} .
$$

Таким образом, автокорреляционная функция поля может быть представлены в виде

$$
g_{1}(\tau)=(1-\rho)\left|g_{1 d}(\tau)\right|+\rho,
$$

где $\rho=I_{s} /\left(I_{d}+I_{s}\right)$ - часть детектируемого света, рассеянного от статичных рассеивающих частиц.

В свою очередь, автокорреляционная функция интенсивности принимает вид

$$
g_{2}(\tau)=1+\beta\left[(1-\rho)\left|g_{1 d}(\tau)\right|+\rho g_{1}(0)\right]^{2} .
$$


Тогда, принимая во внимание уравнения (6)-(8), спеклконтраст можно представить как

$$
\begin{aligned}
K(T)= & {\left[\frac{2 \beta}{T} \int_{0}^{T}\left[\frac{(1-\rho)\left|g_{1 d}(\tau)\right|+\rho g_{1}(0)}{g_{1}(0)}\right]^{2}\right.} \\
& \left.\times\left(1-\frac{\tau}{T}\right) d \tau\right]^{1 / 2} .
\end{aligned}
$$

В случае полностью динамической среды уравнение (9) упрощается до уравнения (5) вследствие отсутствия статичных рассеивающих частиц $(\rho \rightarrow 0)$. Тем не менее в случае статически неподвижной рассеивающей свет среды уравнение (9) не упрощается до постоянного значения спекл-контраста, как это можно было бы ожидать из уравнения (1).

Несмотря на существенные ограничения применимости метода ЛСКВ в условиях нарушения эргодичности, были предложены различные методы. В частности, эффективными решениями являются мультиэкспозиционная ЛСКВ [27] и использование медленно вращающегося диффузора в оптической установке [26] .

Важно отметить, что термины „эргодичность“ и „неэргодичность“ в данной работе рассматриваются в рамках конечного времени экспозиции камеры. Эргодичность в общем смысле подразумевает наблюдение объекта в течение неограниченного времени. В экспериментальных же условиях время одиночного измерения ограничено временем экспозиции камеры, и полученное изображение представляет из себя усредненный сигнал. Более того, в большинстве случаев время экспозиции камеры значительно больше, чем время релаксации динамической компоненты спекл-структуры, но меньше, чем время релаксации для статичной. Таким образом, наблюдаемая среда может быть классифицирована как эргодичная или неэргодичная в зависимости от времени экспозиции камеры. Например, очень медленный поток является эргодичным процессом в общем смысле, поскольку временная и пространственная статистики будут одинаковы в случае неограниченного времени наблюдения. Тем не менее при записи на камеру с очень коротким временем экспозиции данный процесс будет классифицирован как неэргодичный [23].

Целью данной работы было исследование границы применимости метода ЛСКВ при невыполнении условия эргодичности, а также оценка оптимальных параметров для проведения измерений на живых обьектах. В этой связи в работе проведено исследование по определению влияния неподвижных структурных включений в неоднородных сильно рассеивающих свет средах, таких как биоткани, на результаты спекл-контрастных измерений с использованием временного и пространственного методов обработки изображений методом ЛСКВ. В заключение в рамках данной работы метод ЛСКВ был опробован в эспериментах транскраниальной визуализации мозга мыши in vivo.

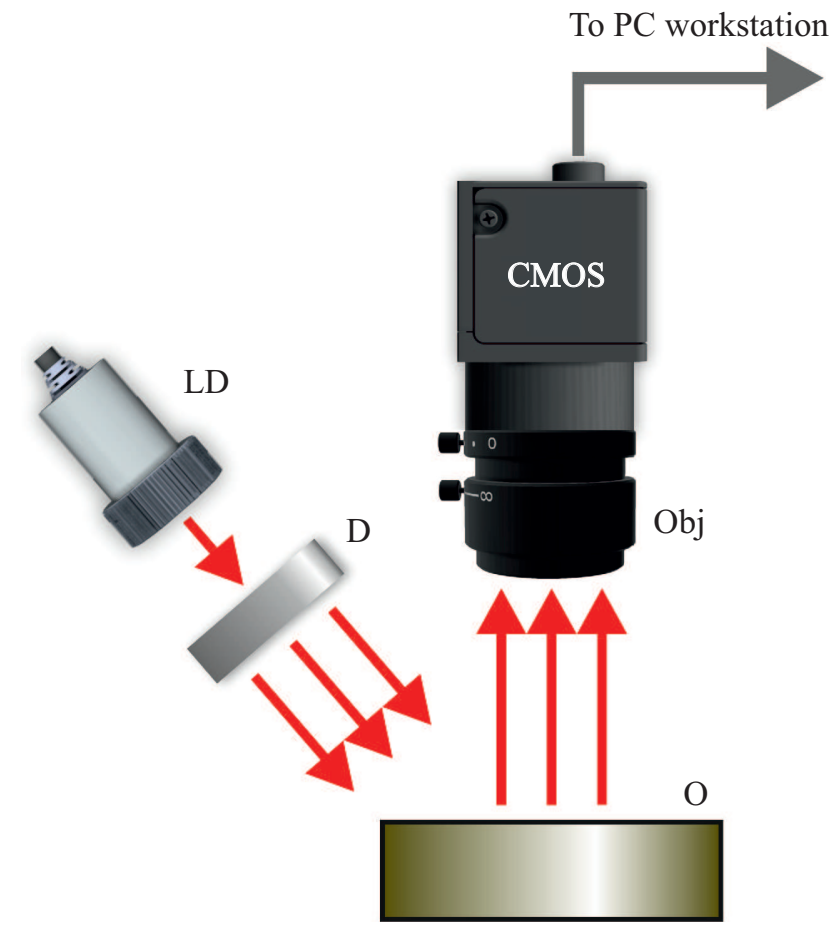

Рис. 1. Оптическая схема экспериментальной установки ЛСКВ. CMOS - КМOП камера; LD - лазерный диод; $\mathrm{D}$ - диффузор; Obj - объектив; O - объект.

\section{Материалы и методы}

На рис. 1 представлена оптическая схема экспериментальной установки, использовавшейся в данной работе. В качестве источника излучения для эксперимента с фантомом использовался лазерный диод на длине волны $655 \mathrm{~nm}$ с мощностью $13 \mathrm{~mW}$ (RLD650-13-3, Roithner Lasertechnik $\mathrm{GmbH}$, Австрия). В качестве источника излучения для эксперимента с мышью использовался лазерный диод на длине волны $808 \mathrm{~nm}$ с мощностью $3 \mathrm{~mW}$ (LDM808/3LJ, Roithner Lasertechnik GmbH, Австрия). Для равномерного распределения лазерного излучения по исследуемому объекту использовался диффузор (ED1-C20, Thorlabs, CША). Для регистрирования спеклструктуры использовалась КМОП камера (DCC3240M, $1280 \times 1024$, размер пикселя $6.7 \mu \mathrm{m}$, Thorlabs, США) совместно с $12 \mathrm{~mm}$ F1.4 объективом (Kenko Tokina Co., Ltd, Япония). Полученные изображения обрабатывались при помощи индивидуально разработанного алгоритма на базе программного обеспечения MATLABr2019b.

Наиболее распространенным методом обработки изображений ЛСКВ является пространственный алгоритм анализа статистики спеклов [28]. Для реализации данного алгоритма статистика спеклов рассчитывается в пределах „окна“, скользящего вдоль исходного спекл-изображения. Как правило, для достижения оптимального результата используется окно размером $5 \times 5$ или $7 \times 7$ пикселей. Основным недостатком пространственного алгоритма является потеря пространствен- 
ного разрешения, определяемая размером скользящего окна. Для решения данной проблемы был предложен альтернативный алгоритм временного анализа статистики спеклов [29]. Для данного алгоритма регистрируется серия последовательных спекл-изображений. Далее статистика спеклов рассчитывается от изображения к изображению по каждому отдельно взятому пикселю серии. Таким образом может быть сохранено пространственное изображение, но потеряно временное. На практике обычно используется комбинация обоих методов с параметрами, подобранными в зависимости от цели исследования [30].

В случае рассеяния света на динамических частицах временной и пространственный алгоритмы показывают одинаковый результат, поскольку выполняется условие эргодичности. Для случая рассеяния от статически неподвижных рассеивателей результат пространственной и временной обработки спекл-изображений оказывается разным. В частности, для идеально сформированной статичной спекл-структуры теоретическое значение пространственного спекл-контраста будет равняться 1, поскольку пространственные флуктуации интенсивности (темные и светлые спеклы) принимают, соответственно, свои минимальные и максимальные значения интенсивности. В данном случае средняя интенсивность будет равна стандартному отклонению интенсивности. Временной спекл-контраст должен равняться 0, поскольку стандартное отклонение в каждом пикселе для серии изображений принимает значение 0 для статичной спекл-структуры. На практике не представляется возможным добиться идеальных условий, поэтому спеклконтраст принимает значения между 0 и 1 . Тем не менее описанная разница между временным и пространственным спекл-контрастом в случае присутствия статически неподвижных рассеивателей может быть значительной и приводить к систематической ошибке в интерпретации полученных результатов.

В предыдущей работе [24] был введен коэффициент спекл-динамики, который позволяет оценить влияние неэргодичной составляющей на результат измерений при помощи ЛСКВ:

$$
C S D=\frac{2 K_{t}}{K_{s}+K_{t}}
$$

где $K_{t}-$ спекл-контраст, рассчитанный при помощи временного алгоритма, а $K_{s}-$ спекл-контраст, рассчитанный при помощи пространственного алгоритма. Теоретически коэффициент принимает значение 1 для динамически рассеивающей свет среды и 0 для мутной среды статически неподвижных рассеивателей. Тем не менее на практике, поскольку $K_{t}$ и $K_{s}$ принимают значения между 0 и $1, C S D$ также не достигает минимальных и максимальных теоретических значений.

Для модельного эксперимента был изготовлен прямоугольный фантом из силикона с отверстием (1 cm в диаметре) на поверхности [31]. В качестве динамических
Оптические параметры липкой ленты и интралипида на длине волны $655 \mathrm{~nm} . \mu_{s}$ - коэффициент рассеяния, $\mu_{a}$ - коэффициент поглощения, $g-$ фактор анизотропии, $n-$ показатель преломления, $\mu_{s}^{\prime}-$ редуцированный коэффициент рассеяния

\begin{tabular}{c|c|c|c|c}
\hline \multicolumn{5}{c}{ Липкая лента } \\
\hline$\mu_{s}, \mathrm{~mm}^{-1}$ & $\mu_{a}, \mathrm{~mm}^{-1}$ & $g$ & $n$ & $\mu_{s}^{\prime}, \mathrm{mm}^{-1}$ \\
\hline 36.38 & 0 & 0.946 & 1.6 & 1.96 \\
\hline \multicolumn{5}{c}{ Интралипид, $3 \%$} \\
\hline$\mu_{s}$ & $\mu_{a}$ & $g$ & $n$ & $\mu_{s}^{\prime}$ \\
\hline 11.8 & 0.02 & 0.55 & 1.33 & 5.31
\end{tabular}

рассеивателей использовался 3\% раствор интралипида. В качестве статичных слоев использовалась непрозрачная липкая лента скотч толщиной $55 \pm 2 \mu \mathrm{m}$. Для расчета изображений коэффициента спекл-динамики фантома детектировались 49 последовательных кадров при времени экспозиции камеры $10 \mathrm{~ms}$. Эти кадры были использованы для расчета временного спекл-контрастного изображения. Также были рассчитаны 49 пространственных спекл-контрастных изображений при размере скользящего окна $7 \times 7$ пикселей. Далее рассчитывались 49 изображений коэффициента спекл-динамики, которые впоследствии были усреднены в финальное изображение. Пространственные спекл-контрастные изображения также были усреднены в одно финальное изображение.

Для транскраниальной визуализации мозга мыши использовалась CD1 мышь женского пола возрастом 8 недель, предоставленная Envigo (Великобритания). В качестве анестезии вводилась внутрибрюшная инъекция $10 \mathrm{mq} / 100 \mathrm{mq} / \mathrm{kq}$ кетамина (Vetoquinol, Франция). После введения анестезии была удалена кожа на лобной, височной, затылочной и теменной областях. Далее исследуемая область головы увлажнялась физиологическим раствором. После этого мышь помещалась на согревающую пластину для поддержания температуры тела $37^{\circ} \mathrm{C}$. Общая продолжительность измерений не превышала $1.5 \mathrm{~h}$, после чего осуществлялась эвтаназия животного при помощи передозировки барбитуратом. Для расчета изображений коэффициента спекл-динамики головы мыши детектировалась последовательность кадров различного размера при времени экспозиции $10 \mathrm{~ms}$. Далее, эти кадры были использованы для расчета временного спекл-контрастного изображения по такому же принципу как и в эксперименте с фантомом.

\section{Результаты и их обсуждение}

Для демонстрации влияния увеличения толщины слоя статически неподвижных рассеивателей на результаты измерений метода ЛСКВ был проведен следующий эксперимент. Отверстие в фантоме заполнялось раствором 
0 films
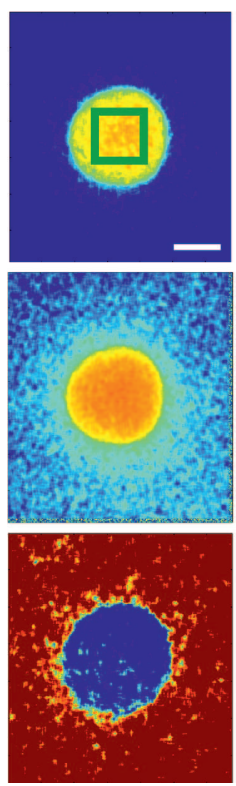

1 films
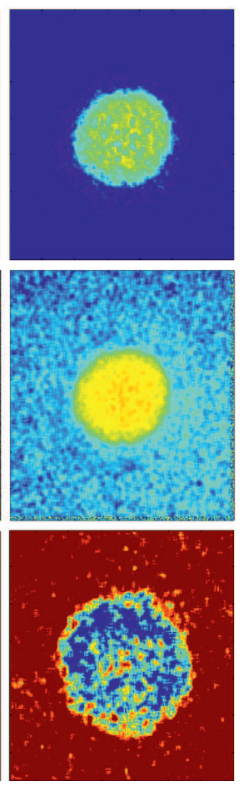

4 films
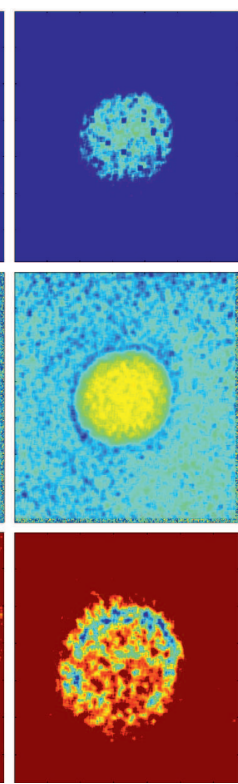

7 films
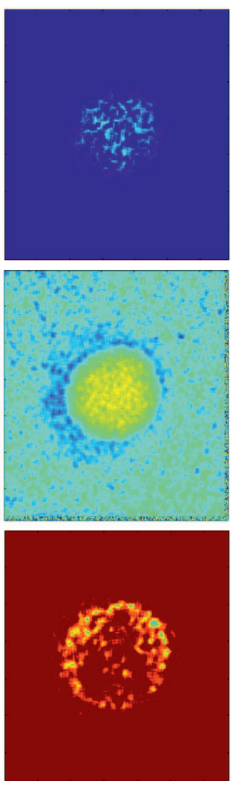

10 films
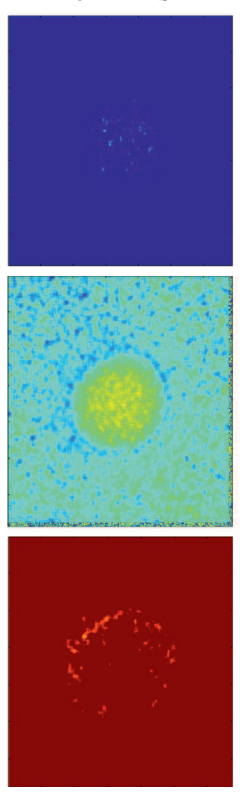

13 films

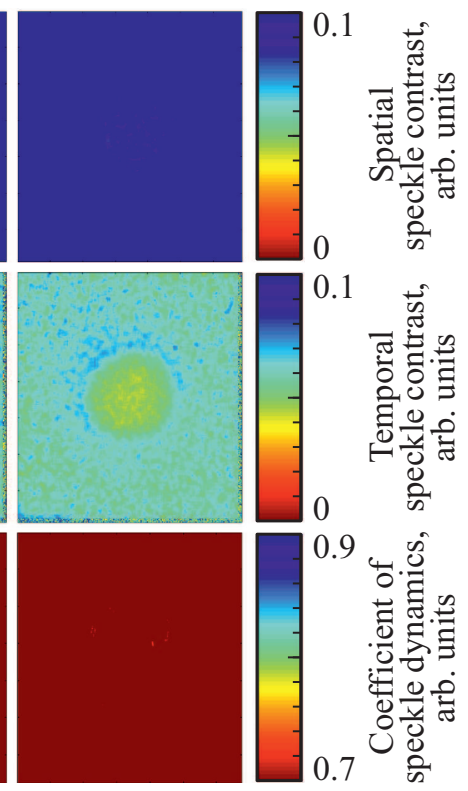

Рис. 2. Пространственные спекл-контрастные изображения (верхняя строка), временные спекл-контрастные изображения и изображения коэффициента спекл-динамики, рассчитанные для эксперимента с фантомом, заполненным раствором интралипида без статичного слоя (0 films) на поверхности, и с 1, 4, 7, 10 и 13 статичными слоями на поверхности. Длина масштабной линии равна $5 \mathrm{~mm}$.

интралипида. На поверхность фантома последовательно один за одним наклеивались слои непрозрачной липкой ленты. Добавление каждого слоя липкой ленты можно рассматривать как увеличение слоя статически неподвижных рассеивателей над динамической рассеивающей свет средой (раствор интралипида). Всего в ходе эксперимента было добавлено 16 слоев, что суммарно составляет толщину $880 \mu \mathrm{m}$. В таблице приведены оптические свойства раствора интралипида [32] и липкой ленты, использовавшиеся для моделирования. Для липкой ленты оптические свойства были измерены экспериментально методом интегрирующей сферы [33].

На рис. 2 приведены примеры пространственных и временных спекл-контрастных изображений, а также изображений коэффициента спекл-динамики, рассчитанных для различного количества слоев статически неподвижных рассеивателей на поверхности фантома. На рис. 3 приведены графики зависимости, а также интерполяционные кривые для среднего значения временного и пространственного спекл-контраста, а также среднего значения коэффициента спекл-динамики, рассчитанных в пределах области, обозначенной зеленым квадратом на рис. 2 для описанного эксперимента.

Из рис. 2 и 3 видно, что интенсивность броуновского движения частиц раствора интралипида остается практически неизменной, в то время как значения пространственного и временного спекл-контраста увеличиваются линейно при последовательном добавлении слоев липкой ленты. Причем, увеличение значений пространственного спекл-контраста происходит в 4 раза быстрее в сравнении с временным. Таким образом, значение спекл-контраста может зависеть не только от скорости движения рассеивающих частиц, но и от толщины слоя статически неподвижных рассеивателей над динамической рассеивающей свет средой. Данный эффект обычно игнорируется в научных работах, использующих метод ЛСКВ. Тем не менее влияние толщины статически неподвижных рассеивателей на значение спекл-контраста должно учитываться особенно при измерениях на биологических объектах, кровеносные сосуды в которых располагаются на различных глубинах. Различное значение спекл-контраста может свидетельствовать не только о скорости кровотока в сосудах, но и об их глубине залегания, что в свою очередь может оказаться причиной систематической ошибки при анализе и интерпретации экспериментальных данных, получаемых методом ЛСКВ. Расчет коэффициента спекл-динамики позволяет отличить изменения значения спекл-контраста в связи с увеличением или уменьшением интенсивности движения рассеивателей от изменения значения спеклконтраста в связи с наличием статически рассеивающего слоя над динамической рассеивающей свет средой [24].

Из рис. 2 и 3 также видно, что в отсутствие статичного рассеивающего слоя над раствором интралипида, пространственный и временной спекл-контраст принимают близкие значения, что свидетельствует о том, что рассматриваемая система удовлетворяет условию эргодичности. При добавлении статичных слоев разница между пространственным и временным спекл-контрастом увеличивается. Также уменьшается значение коэффициента 


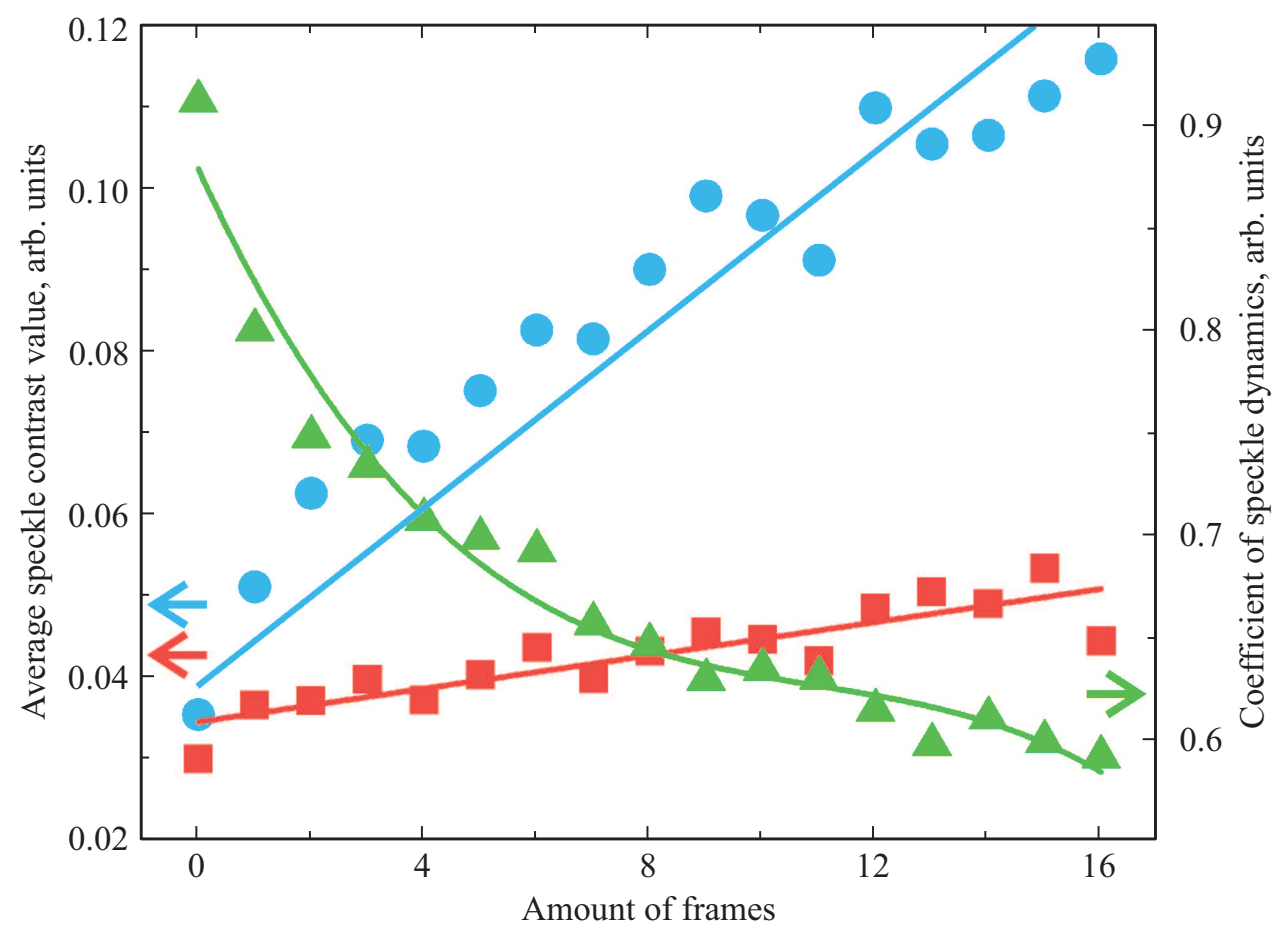

Рис. 3. График зависимости среднего значения пространственного спекл-контраста (синие круги), среднего значения временного спекл-контраста (красные квадраты) и среднего значения коэффициента спекл-динамики (зеленые треугольники) от количества статичных слоев на поверхности фантома. Символами обозначены экспериментально измеренные значения. Линиями обозначены интерполяционные кривые.

спекл-динамики, что свидетельствует о росте нарушений в условии эргодичности. Данные нарушения являются причиной возникновения систематической ошибки в методе ЛСКВ и должны надлежащим образом учитываться при измерении на биологических объектах. Из рис. 3 видно, что значение временного спекл-контраста подвергается меньшему влиянию при увеличении толщины статичного слоя. Более того, при временной обработке область, заполненная интралипидом, остается видна даже при наличии большого количества статических рассеивающих слоев. Таким образом, количественная оценка скорости кровотока в сосудах может сильно зависеть от выбранного метода обработки спекл-изображений. В то же время качественные измерения, при которых важно показать лишь относительное изменение кровотока или перфузии, практически не будут страдать от несоответствия рассматриваемой системы условию эргодичности. Из рисунков также видно, что значение коэффициента спекл-динамики снижается обратно пропорционально увеличению доли статически неподвижных рассеивателей (неэргодической составляющей) (рис. 2 и 3). Можно предположить, что наличие статичных слоев с небольшой толщиной вносит незначительную ошибку в оценку спекл-контраста. Таким образом, коэффициент спеклдинамики можно использовать для оценки влияния заглубленности сосуда на результат спекл-контрастных измерений.
Оценка эффективного измеряемого объема в модельных экспериментах с использованием фантомов с различным количеством статичных слоев осуществлялась методом Монте-Карло [34]. На рис. 4 представлены результаты моделирования эффективного измеряемого объема, рассчитанного для модельного эксперимента с использованием фантома с различным количеством статичных слоев. Оптические свойства, представленные в таблице, соответствуют излучению на длине волны $655 \mathrm{~nm}$. Для оценки эффективного измеряемого объема ткани, не зависящего от длины волны, смоделированные данные были построены в зависимости от оптической плотности, которая рассчитывалась как толщина слоя $(D)$ умноженная на редуцированный коэффициент рассеяния $\left(\mu_{s}^{\prime}\right)$. Из рисунка видно, что значительный эффективный объем излучения от интралипида регистрируется детектором даже в присутствии нескольких статичных слоев (вплоть до 7 слоев липкой ленты, что суммарно составляет $385 \mu \mathrm{m}$ ), что позволяет проводить более или менее точные измерения при ЛСКВ.

Типичная толщина черепа мыши возрастом до 2 месяцев составляет по разным источникам от 150 до $310 \mu \mathrm{m}[35,36]$, а инфракрасное излучение характеризуется большой глубиной проникновения в биологические ткани. Редуцированный коэффициент рассеяния в инфракрасной области для черепа мыши составляет $1.75 \pm 0.05$ [35]. Таким образом, наличие статичного рассеивающего слоя в виде костей черепа не вносит 


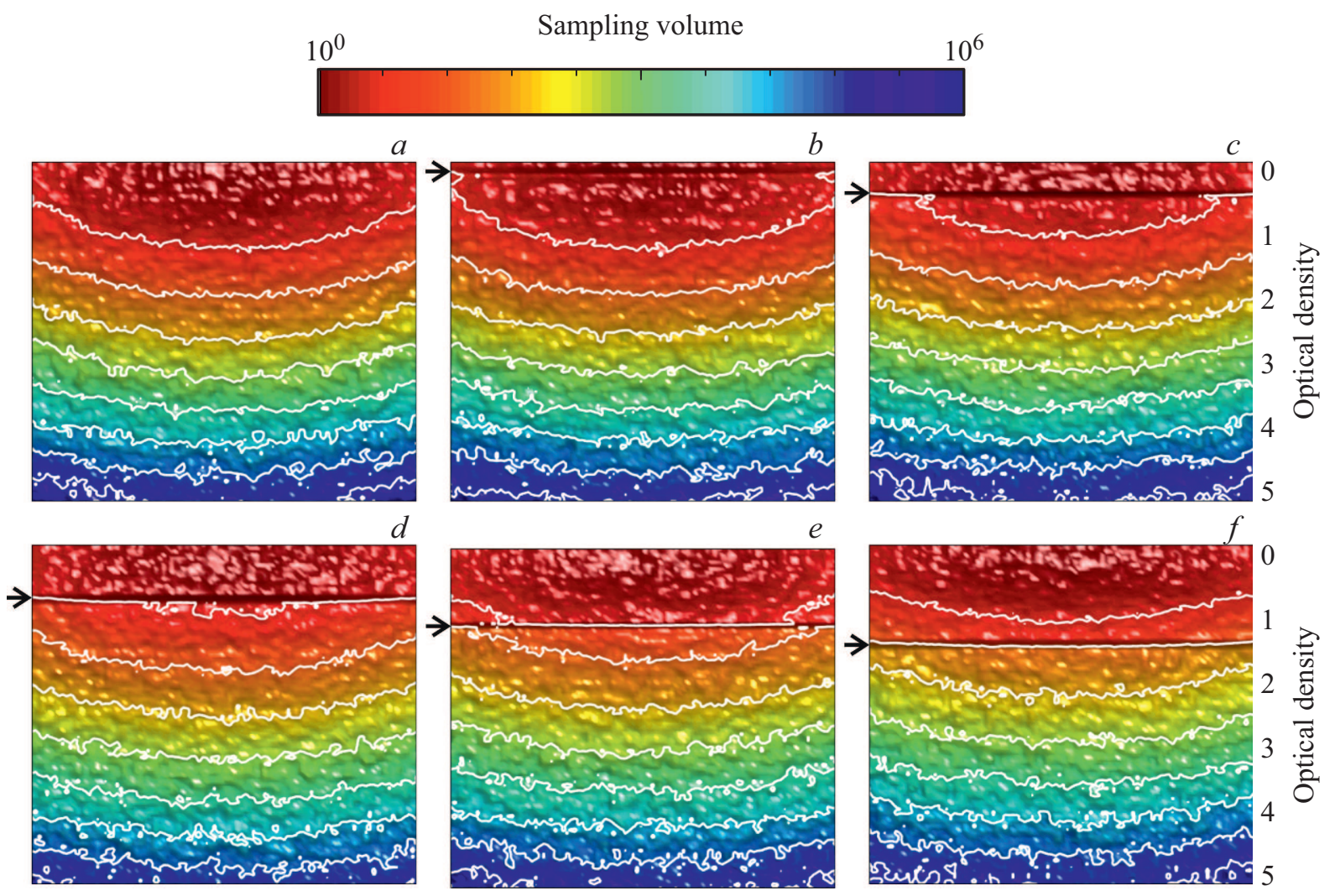

Рис. 4. Моделирование эффективного объема регистрации излучения методом Монте-Карло для интралипида в отсутствие статичного слоя над ним $(a)$, для интралипида с $1(b), 4(c), 7(d), 10(e)$ и $13(f)$ статичными слоями над ним. Черной стрелкой изображено положение нижней границы статичного слоя.

существеных искажений в результаты измерения кровотока мозга мыши методом ЛСКВ. На рис. 5 изображены примеры пространственных и временных спеклконтрастных изображений, а также изображений коэффициента спекл-динамики, рассчитанных с использованием различной последовательности исходных спеклизображений при транскраниальной визуализации кровотока в мозге мыши in vivo. Как уже было отмечено выше, толщина черепа молодой мыши не вносит существенных ошибок в результаты измерений. Из рис. 5 хорошо видно, что при обработке с использованием последовательности из 10 кадров пространственное и временное спекл-контрастные изображения одинаковы как для областей мозга, соответствующих кровеносным сосудам (точка 2 на рис. 5), так и для областей без сосудов (точка 1 на рис. 5). Также коэффициент спекл-динамики имеет высокое значение в областях измерения, соответствующих мозгу, что свидетельствует о том, что условия эргодичности не нарушены или незначительны. Тем не менее ЛСКВ основывается на статистических измерениях, поэтому важно учитывать размер выборки данных, что в данном случае является количеством последовательных кадров, используемых для получения спекл-контрастных изображений. Из рис. 5 видно, что уменьшение количества кадров сильно влияет на результат временной обработки. В частности, увеличивается разница между пространственными и временными спекл-контрастными изображениями как для областей мозга, соответствующих наличию кровотока/кровеносных сосудов (точка 2 на рис. 5), так и их отсутствия (точка 1 на рис. 5), что также хорошо видно из изображения коэффициента спекл-динамики. Данный эффект также требуется учитывать при использовании пространственно-временного алгоритма обработки спекл-изображений. В нашем случае в эксперименте с временем экспозиции $10 \mathrm{~ms}$ было установлено, что минимально достаточная выборка последовательных исходных изображений равна 10 кадрам.

Неверно подобранные время экспозиции камеры, алгоритмы обработки спекл-изображений, количество кадров, используемых для обработки изображений, а также наличие рассеивающего статичного слоя могут существенно влиять на результаты измерений методом ЛСКВ. Оптимальные параметры ЛСКВ должны подбираться исходя из того, нужно ли получить количественные или же качественные данные, важнее ли сохранить пространственное разрешение или временное и т.д. Коэффициент спекл-динамики позволяет оценить влияние неэргодичности системы при заданном времени экспозиции, что может помочь подобрать оптимальные 

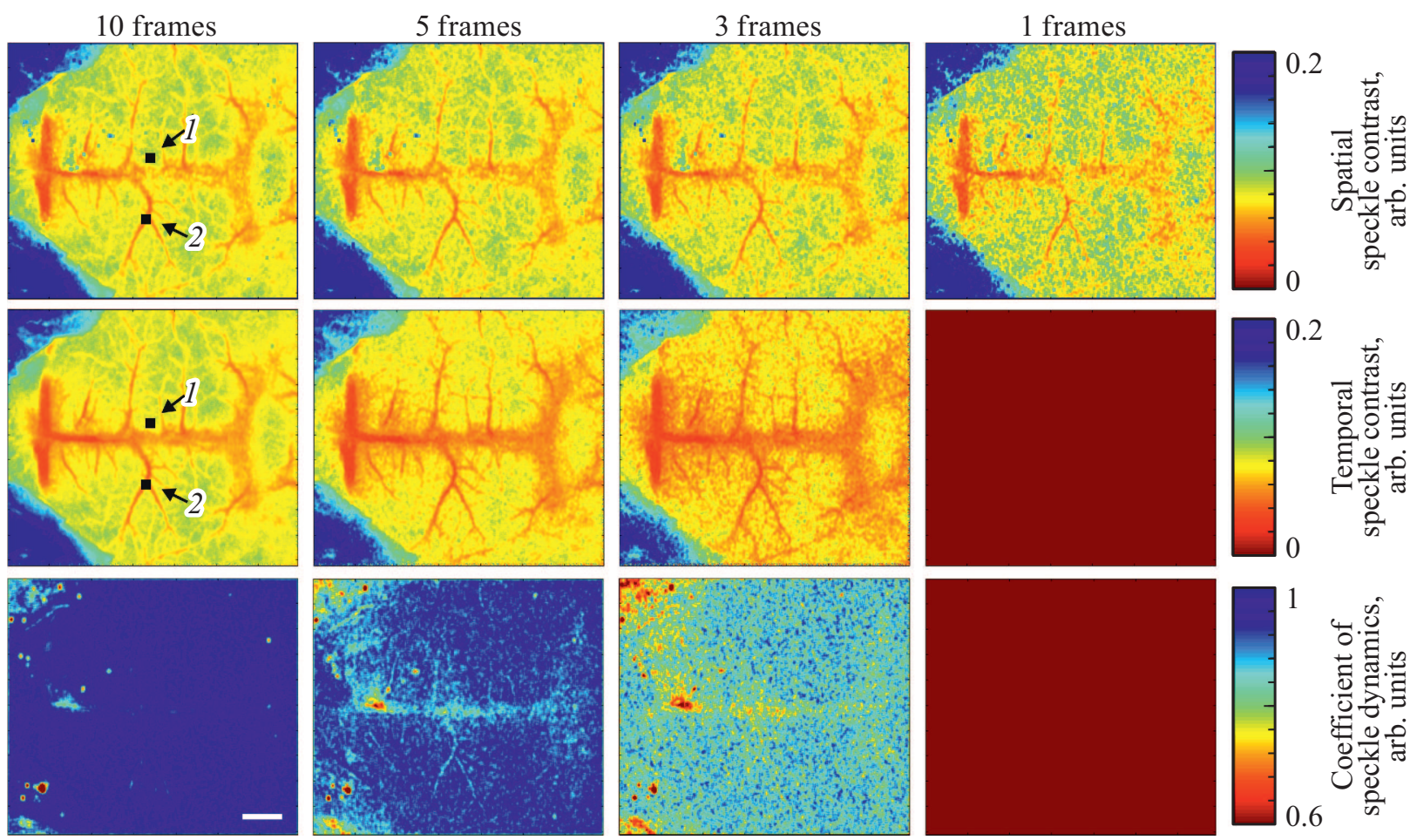

Рис. 5. Пространственные спекл-контрастные изображения (верхняя строка), временные спекл-контрастные изображения и изображения коэффициента спекл-динамики, рассчитанные для транскраниальной визуализации мозга мыши с использованием 10 , 5, 3 и 1 последовательных спекл-изображений. Длина масштабной линии равна $1 \mathrm{~mm}$.

параметры для ЛСКВ измерений. В частности, в данной работе было показано, что кости черепа при визуализации кровотока мозга мыши не вносят существенных искажений на выполнимость условия эргодичности. Тем не менее для минимизации систематической ошибки ЛСКВ эксперимента требуется удаление кожного покрова с области измерений. В случае взрослой особи мыши помимо удаления кожи проводится также либо процедура утоньшения черепа мыши с помощью дрели [37], либо его полное удаление [38]. Использование метода оптического просветления [39] потенциально может сократить влияние слоя статически неподвижных рассеивателей биоткани (в особенности кожи [40]) на результаты ЛСКВ, тем самым увеличив эффективность неинвазивной визуализации кровотока мозга in vivo.

\section{Заключение}

В данной работе было изучено влияние изменения условия эргодичности наблюдаемой неоднородной сильно рассеивающей свет среды на результаты ЛСКВ. Было экспериментально показано на фантоме, что увеличение в измеряемом объеме числа неподвижных рассеивателей по отношению к динамическим вносит разницу в результаты пространственной и временной обработки спекл-изображений при заданном времени экспозиции. Анализ полученных экспериментальных значений про- странственного и временного спекл контраста, значений коэффициента спекл-динамики, а также анализ эффективного объема регистрации излучения смоделированного методом Монте-Карло позволил предположить, что наличие относительно тонкого, до $30 \%$ от общего объема, статичного слоя не вносит значительных изменений в результаты ЛСКВ. Данное предположение было подтверждено с помощью транскарниальной in vivo визуализации кровотока мозга мыши возрастом 8 недель. Было показано, что нарушение условия эргодичности в наблюдаемом объекте должно учитываться при измерениях. Также, было показано, что параметры системы для ЛСКВ, такие как время экспозиции камеры, метод анализа спекл-изображений, а также количество кадров, используемых для обработки, должны подбираться индивидуально для каждого эксперимента в соответствии с поставленными задачами.

\section{Финансирование работы}

А.С. выражает благодарность гранту Финского культурного фонда (Finnish Cultural Foundation grant) № 00180998. И.М. выражает благодарность за частичную поддержку грантам Академии Финляндии (Academy of Finland) № 326204 и № 325097, проекту NEUROPA № 863214 по программе исследований и инноваций Европейского Союза Horizon 2020, проекту Академического Превосходства МИФИ (MEPhI Academic Excellence 
Project) № 232 02.a03.21.0005 и программе финансирования Национального исследовательского Томского государственного университета им. академика Д.И. Менделеева. А.Б. выражает благодарность гранту Академии Финляндии (Academy of Finland) № 290596. А.П. выражает благодарность гранту Академии Финляндии (Academy of Finland) № 314369 и проекту INFOTECH. В.К. выражает благодарность грантовой программе для научных сотрудников - The Henry Chanoch Krenter Institute for Biomedical Imagin gand Genomics (Staff Scientists grant program).

\section{Соблюдение этических норм}

Исследование на животном было одобрено Комитетом по уходу и использованию животных института естественных наук Вейцмана (Weizmann Institute of Science Institutional Animal Care and Use committee (IACUC)). Все применимые институциональные принципы ухода и использования животного были соблюдены.

\section{Конфликт интересов}

Авторы заявляют, что у них нет конфликта интересов.

\section{Список литературы}

[1] Leahy M.J. Microcirculation Imaging. John Wiley \& Sons, 2012.

[2] Меглинский И.В., Кальченко В.В., Кузнецов Ю.Л., Кузник В.И., Тучин В.В. // Докл. АН. 2013. Т. 451. № 4. C. 393; Meglinski I.V., Kalchenko V.V., Kuznetsov Y.L., Kuznik B.I., Tuchin V.V. // Dokl. Phys. 2013. V. 58. N 8. P. 323. doi 10.1134/S102833581308003X

[3] Kalchenko V., Kuznetsov Y., Harmelin A., Meglinski I.V. // J. Biomed. Opt. 2012. V. 17. N 5. P. 050502. doi 10.1117/1.JBO.17.5.050502

[4] Кальченко В.В., Кузнецов Ю.Л., Меглинский И.В. // Квант. электрон. 2013. Т. 43. № 7. С. 679; Kalchenko V., Kuznetsov Y.L., Meglinski I. // Quantum Electron. 2013. V. 43. N 7. P. 679. doi 10.1070/QE2013v043n07ABEH014953

[5] Kalchenko V., Kuznetsov Y.L., Preise D., Meglinski I., Harmelin A. // J. Biomed. Opt. 2014. V. 19. N 6. P. 060502. doi 10.1117/1.JBO.19.6.060502

[6] Kalchenko V., Meglinski I., Sdobnov A., Kuznetsov Y., Harmelin A. // J. Biomed. Opt. 2019. V. 24. N 6. P. 060501. doi 10.1117/1.JBO.24.6.060501

[7] Kalchenko V., Israeli D., Kuznetsov Y.L., Meglinski I., Harmelin A. // J. Biophotonics. 2015. V. 8. N 11-12. P. 897. doi 10.1002/jbio.201400140

[8] Kalchenko V., Sdobnov A., Meglinski I., Kuznetsov Y., Molodij G., Harmelin A. // Photonics. 2019. V. 6. N 3. P. 80. doi 10.3390/photonics6030080

[9] Mizeva I., Dremin V., Potapova E., Zherebtsov E., Kozlov I., Dunaev A. // IEEE Trans. Biomed. Eng. 2019. doi 10.1109/TBME.2019.2950323

[10] Goodman J.W. Laser Speckle and Related Phenomena / Ed. by Dainty J.C., V. 9 in series Topics in Applied Physics. Springer, Berlin, Heidelberg, 1975. P. 9.
[11] Goodman J.W. Speckle Phenomena in Optics: Theory and Applications. Roberts and Company Publishers, 2007.

[12] Fercher A., Briers J. // Opt. Commun. 1981. V. 37. N 5. P. 326. doi 10.1016/0030-4018(81)90428-4

[13] Boas D., Yodh A.G. // J. Opt. Soc. Amer. A. 1997. V. 14. N 1. P. 192. doi 10.1364/JOSAA.14.000192

[14] Cummins H. Photon Correlation and Light Beating Spectroscopy. Springer Science \& Business Media, 2013. V. 3.

[15] Berne B.J., Pecora R. Dynamic Light Scattering: with Applications to Chemistry, Biology, and Physics. Courier Corporation, 2000.

[16] Parthasarathy A.B., Tom W.J., Gopal A., Zhang X., Dunn A.K. // Opt. Exp. 2008. V. 16. N 3. P. 1975. doi 10.1364/OE.16.001975

[17] Davenport W.B., Root W.L. An Introduction to the Theory of Random Signals and Noise. N. Y.: McGraw-Hill, 1958. V. 159.

[18] Yaglom A.M. An Introduction to the Theory of Stationary Random Functions. Courier Corporation, 2004.

[19] Zakharov P., Volker A., Buck A., Weber B., Scheffold F. // Opt. Lett. 2006. V. 31. N 23. P. 3465. doi 10.1364/OL.31.003465

[20] Joosten J.G.H., Gelade E.T.F., Pusey P.N. // Phys. Rev. A. 1990. V. 42. N 4. P. 2161. doi 10.1103/PhysRevA.42.2161.

[21] Li P., Ni S., Zhang L., Zeng S., Luo Q. // Opt. Lett. 2006. V. 31. N 12. P. 1824. doi 10.1364/OL.31.001824

[22] Zakharov P. // Opt. Lett. 2017. V. 42. N 12. P. 2299. doi 10.1364/OL.42.002299

[23] Pusey P.N., Van Megen W. // Physica A: Stat. Mech. Appl. 1989. V. 157. N 2. P. 705. doi 10.1016/0378-4371(89)90063-0

[24] Sdobnov A., Bykov A., Molodij G., Kalchenko V., Jarvinen T., Popov A., Kordas K., Meglinski I. // J. Phys. D: Appl. Phys. 2018. V. 51. N 15. P. 155401. doi 10.1088/1361-6463/aab404

[25] Sdobnov A., Bykov A., Popov A., Zherebtsov E., Meglinski I. // Proc. SPIE. 2018. V. 10685. P. 1068509. doi 10.1117/12.2306631

[26] Volker A.C., Zakharov P., Weber B., Buck F., Scheffold F. // Opt. Expr. 2005. V. 13. N 24. P. 9782. doi 10.1364/OPEX.13.009782

[27] Rice T.B., Kwan E., Hayakawa C.K., Durkin A.J., Choi B., Tromberg B.J. // Biomed. Opt. Expr. 2013. V. 4. N 12. P. 2880. doi 10.1364/BOE.4.002880

[28] Boas D.A., Dunn A.K. // J. Biomed. Opt. 2010. V. 15. N 1. P. 011109. doi 10.1117/1.3285504

[29] Cheng H., Luo Q., Zeng S., Chen S., Cen J., Gong H. // J. Biomed. Opt. 2003. V. 8. N 3. P. 559. doi 10.1117/1.1578089

[30] Qiu J., Li P., Luo W., Zhang H., Luo Q. // J. Biomed. Opt. 2010. V. 15. N 1. P. 016003. doi 10.1117/1.3290804

[31] Wrobel M.S., Popov A.P., Bykov A.V., Tuchin M., Jedrzejewska-Szczerskaa M. // Biomed. Opt. Express. 2016. V. 7. N 6. P. 2088. doi 10.1364/BOE.7.002088

[32] Flock S.T., Jacques S.L., Wilson B.C., Star W.M., van Gemert M.J. // Las. Surg. Med. 1992. V. 12. N 5. P. 510. doi 10.1002/1sm.1900120510

[33] Pickering J.W., Prahl S.A., Van Wieringen N., Beek J.F., Sterenborg H.J., Van Gemert M.J. // Appl. Opt. 1993. V. 32. N 4. P. 399. doi 10.1364/AO.32.000399

[34] Meglinski I.V., Matcher S.J. // Opt. Spectrosc. 2001. V. 91. N 4. P. 654. doi 10.1134/1.1412689

[35] Soleimanzad H., Gurden H., Pain F. // J. Biomed. Opt. 2017. V. 22. N 1. P. 010503. doi 10.1117/1.JBO.22.1.010503

[36] Park J.H., Sun W., Cui M. // Proc. Nat. Acad. Sci. 2015. V. 112. N 30. P. 9236. doi 10.1073/pnas.1505939112 
[37] He J., Lu H., Deng R., Young L., Tong S., Jia X. // EMBC Proc. 2015. P. 6971. doi 10.1109/EMBC.2015.7319996

[38] Dunn A.K. // Ann. Biomed. Eng. 2012. V. 40. N 2. P. 367. doi 10.1007/s10439-011-0469-0

[39] Sdobnov A.Y., Darvin M.E., Genina E.A., Bashkatov A.N., Lademann J., Tuchin V.V. // Spectrocim. Acta A. 2018. V. 197. P. 216-229. doi 10.1016/j.saa.2018.01.085

[40] Сдобнов А.Ю., Ладеманн Ю., Дарвин М.Е., Тучи В.В. // Успехи Биол. Хим. 2019. Т. 59. С. 295; Sdobnov A.Y., Lademann J., Darvin M.E., Tuchin V.V. // Biochem. Moscow. 2019. V. 84. N 1. P. 144. doi 10.1134/S0006297919140098 